\title{
Prosthetic Rehabilitation of a Patient with Ameloblastoma using an Unconventional Cast Partial Denture
}

\section{Amanda Nadia Ferreira*, Meena Aras, Vidya Chitre and Kennedy Mascarenhas}

Goa Dental College \& Hospital, India

\begin{abstract}
This case report describes the prosthodontic rehabilitation of a patient with a previous history of follicular ameloblastoma, in the third quadrant which was treated by marginal resection of the mandible and an immediate replacement of missing bone with an iliaccrest bone graft. An unconventional Cast partial denture design was used, due to the complete obliteration of the labial vestibule and the limited amount of mesio-distal space for the placement of the denture teeth
\end{abstract}

Keywords: Prosthodontic; Rehabilitation

\section{Introduction}

The World Health Organization (WHO 2005), classifies ameloblastoma as a benign odontogenic tumor ,that accounts for around $23 \%$ of odontogenic tumors [1], yet only $1 \%$ of all tumors of the maxilla and mandible are ameloblastomas [2-4]. There is a slight male predilection and major occurrence in the mandibular molar-ramus area with a peak incidence in the $3 \mathrm{rd}-4$ th decade of life $[1,5]$.

Ameloblastomas can be classified as solid/multicystic, intraosseous or unicystic, with peripheral subtypes [6,7] even though it is a benign tumor, it is treated aggressively because of the myeline nature of its growth. Primary resection is considered as the only predictable treatment option [7-11]. This leaves the patient with a defect in the affected site and impaired swallowing, speech, saliva, mastication, and cosmesis. The two main treatment modalities available are implant retained fixed prosthesis and removable partial dentures. implant retained prosthesis provides increased retention, stability and support, however it necessitates additional surgical procedures with associated risks, increased rehabilitation period and increased cost. Removable cast partial dentures aim at restoring function within the unique movement capabilities of the resected mandible. The basic prosthodontic design principles should be followed during fabrication of the prosthesis to minimize dislodgement, increase stability and optimize prosthesis function [12]. The rehabilitation of such a patient poses a serious challenge to the prosthodontists as well as the laboratory personnel.

\section{Case Report}

A 53 year old female patient was referred to the department of Prosthodontics for the replacement of missing teeth. The patient's medical/dental history referred to three reoccurrences of a tumor, which was classified as unicystic mural ameloblastoma. The tumor was located in the posterior right mandibular region encompassing six teeth, specifically the lateral incisor, canine, first and second premolars and the first and second molar. Excision of the lesion was carried out followed by the marginal resection of the mandible and immediate placement with an iliac crest bone graft and stabilized with reconstruction miniplates. All six teeth were removed along with the tumor. Intra oral examination revealed several missing teeth $36,42,43,44,45,46,47$. There was reduced mesio-distal space for the replacement of 36 , also a complete obliteration of the labial vestibule in the defect site was seen. 48 was severely lingually tilted. There was a tori in the anterior lingual vestibule (Figure 1).

After extra and intra-oral examination, the treatment plan was discussed with the patient. An implant-retained prosthesis was suggested to the patient however due to financial constraints, the patient opted to carry out the rehabilitation with a cost effective, conventional method. The conventional cast partial denture (CPD) needed to be modified to account for the absence of the vestibular sulcus.

\section{Treatment Procedure}

Preprosthetic treatment included the surgical removal of the torus mandibularis as it would hinder the path of placement and removal of the cast partial denture. Diagnostic impressions were made and diagnostic casts were obtained.The diagnostic cast was surveyed to identify the most desirable path of placement with minimal interferences, distinguish proximal surfaces which will act as guide planes and establish and measure undercuts.

The CPD design included a lingual plate as a major connector ,a ring clasp on 48 to accommodate for the severe lingual tilt. An I bar

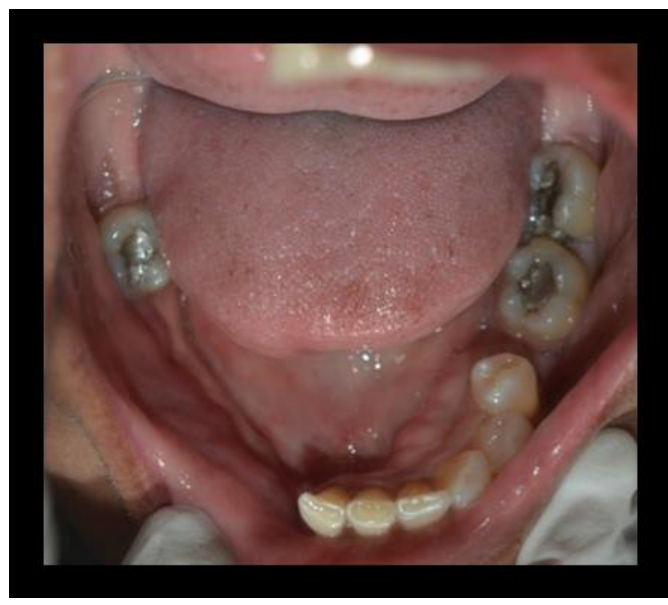

Figure 1: Mandibular dental arch

*Corresponding author: Amanda Nadia Ferreira, Goa Dental College \& Hospital, India, Tel: 0832245 9812; E-mail: Amanda12590@gmail.com

Received April 04, 2018; Accepted April 15, 2018; Published April 22, 2018

Citation: Ferreira AN, Aras M, Chitre V, Mascarenhas K (2018) Prosthetic Rehabilitation of a Patient with Ameloblastoma using an Unconventional Cast Partial Denture. Dentistry 8: 482. doi:10.4172/2161-1122.1000482

Copyright: (c) 2018 Ferreira AN, et al. This is an open-access article distributed under the terms of the Creative Commons Attribution License, which permits unrestricted use, distribution, and reproduction in any medium, provided the original author and source are credited. 
on 42 , an embrassure clasp on 36,37 . A metal pontic with porcelain veneered facially was planned to replace 36 and included in the metal framework due to the limited Mesio-distal space. Vertical struts (4-5 $\mathrm{mm}$ ) were made on the minor connector for additional retention onto which tube teeth were to be fitted, to compensate for the complete obliteration of the buccal vestibule.

Mouth preparations were done for various cast partial components and an impression was made of the mandibular arch with addition silicone (Aquasil, Dentsply) using the two step putty wash technique. The master cast was poured in type IV die stone (Kalabhai) and surveyed to ascertain and check parallelism of guide planes, survey lines and useful undercuts in final designing of the framework. Block out procedure was done and the cast was duplicated in reversible hydrocolloid material and poured with refractory material (Wirovest, BEGO). Wax pattern fabrication using stippled, grid retention and the beading wax (Figure 2). The CPD was cast in a conventional manner (Figure 3). The finished metal framework was tried in the patient's mouth to assess the fit and availability of interarch space (Figure 4). Wax rims were adjusted to record the vertical dimension. Casts were mounted in centric relation. The acrylic teeth were arranged in a mutually protected occlusal scheme. Shade was selected for the denture teeth and the veneering porcelain on 36 . Try in was done and fabricated denture was inserted (Figures 5 and 6).

The radio graphical examination after two years revealed no reoccurrence of the lesion. Clinical examination showed that the patient

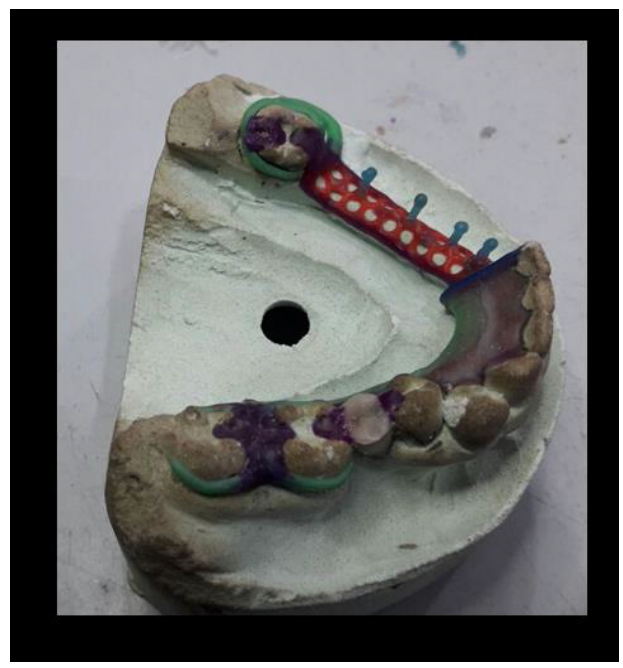

Figure 2: Wax pattern fabricated.

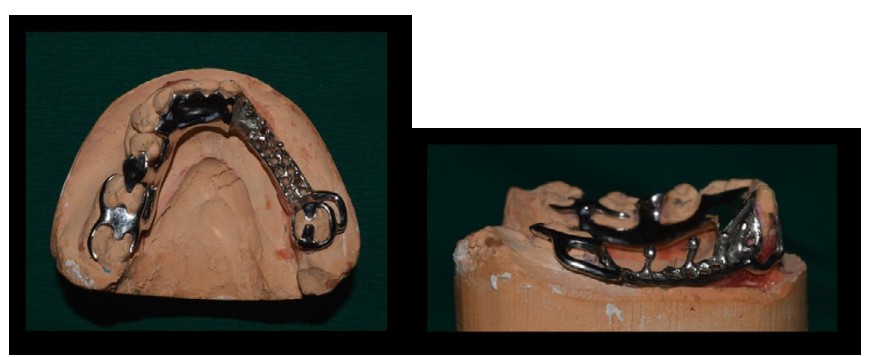

Figure 3: Framework cast in cobalt chromium alloy.

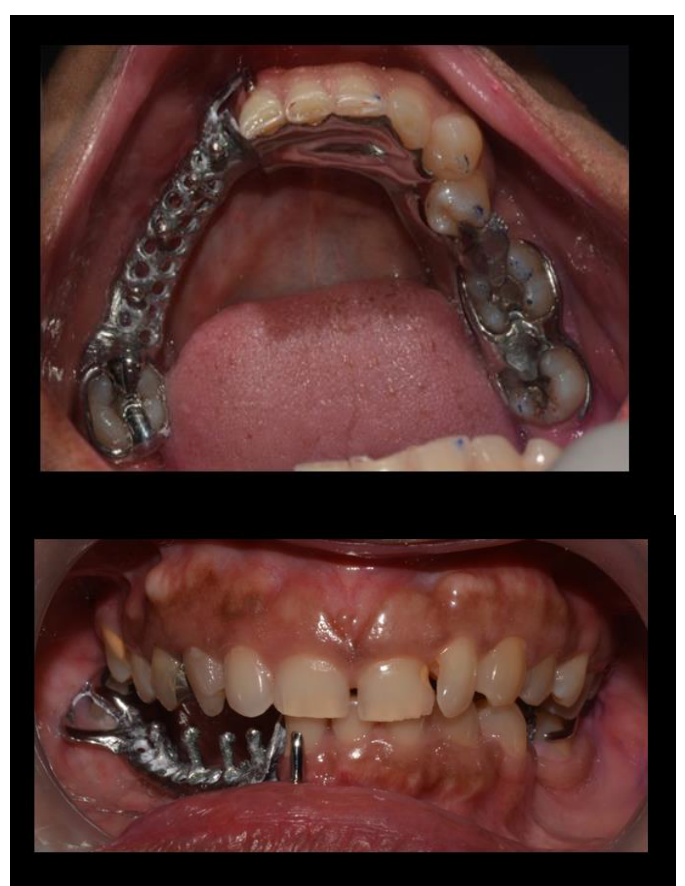

Figure 4: Framework fit verified.

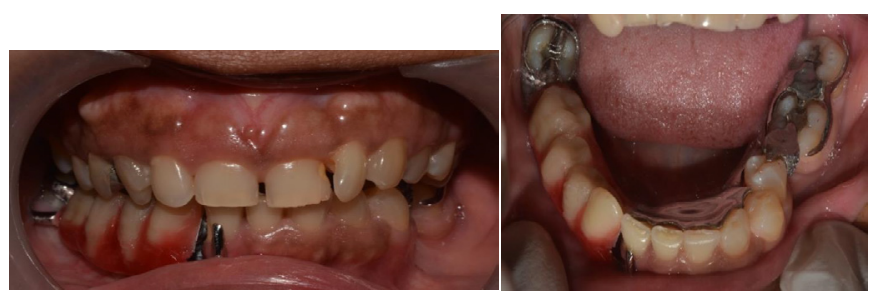

Figure 5: Try in.

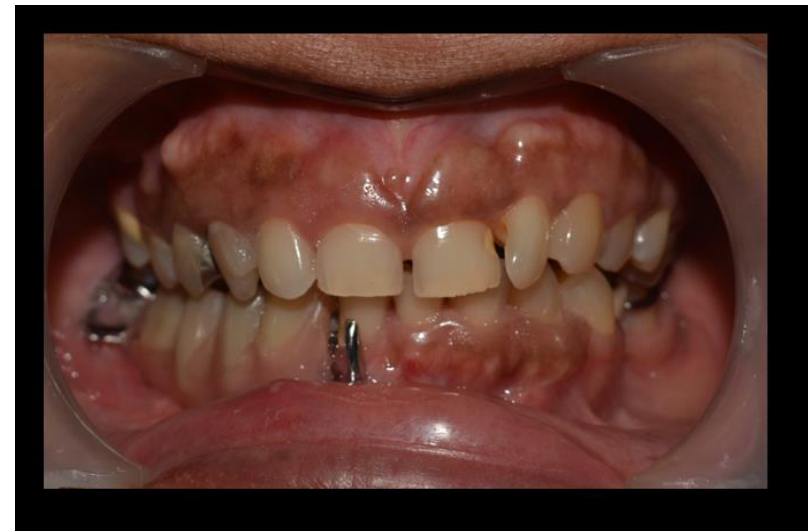

Figure 6: Final CPD insertion.

was maintaining satisfactory oral hygiene. The patient was able to chew well and was extremely satisfied with the results.

\section{Discussion}

The complete treatment of a patient with cancer of the mandible requires an interdisciplinary approach including surgery, radiation 
Citation: Ferreira AN, Aras M, Chitre V, Mascarenhas K (2018) Prosthetic Rehabilitation of a Patient with Ameloblastoma using an Unconventional Cast Partial Denture. Dentistry 8: 482. doi:10.4172/2161-1122.1000482

therapy, chemotherapy, and prosthetic rehabilitation. The extent of surgery could interrupt mandibular continuity and lead to facial disfigurement and mandibular function impairment but maintaining continuity helps preserve normal muscle function and facial contours that leads to better rehabilitation of prosthesis. For this patient, a fixed partial denture (FPD) was not suitable, as it would have resulted in overly long pontics compromising the biomechanics of the prosthesis. An unconventional metal partial denture has a better prognosis as there was an absence of any deviation of the mandible during opening and closing, also, the remaining teeth were in good condition and could serve as abutments. The inclusion of the pontic in the CPD design, and the incorporation of the ring clasp on the severely tilted mandibular molar, was a conservative approach as it prevented the need for intentional root canal therapy and tooth preparation of the abutment teeth. The vertical struts included in the minor connector design were used to support the denture bases and account for the limited vestibular sulcus as a result of mandibular resection on the right side.

\section{References}

1. de Moraes FB, Cardoso RM, Rodrigues SV, Dutra MV, Pereira UR, et al. (2014) Ameloblastoma: A clinical and therapeutic analysis on six cases. Rev Bras Ortop 49: 305-308.

2. Gorlin RJ, Chaudhry AP, Pindborg JJ (1961) Odontogenic tumors: Classification, histopathology clinical behaviour in man and domesticated animals. Cancer 14 73-101.

3. Adekeye EO (1980) Ameloblastoma of jaws: A survey of 109 Nigerian patients. J Oral Surg 38: 36-41.

4. Adekeye EO, Lavery KM (1986) Recurrent ameloblastoma of the maxillofacial region. Clinical features and treatment. J Maxillofac Surg 14: 153-157.

5. Krishnapillai R, Angadi PV (2010) A clinical, radiographic, and histologic review of 73 cases of ameloblastoma in an Indian population. Quintessence Int 41: 90-100.

6. Ghandhi D, Ayoub AF, Pogrel MA, MacDonald G, Brocklebank LM, et al. (2006) Ameloblastoma: a surgeon's dilemma. J Oral Maxillofac Surg 64: 1010-1014.

7. Carlson ER, Marx RE (2006) The ameloblastoma: primary, curative surgical management. J Oral Maxillofac Surg 64: 484-494.

8. Peterson LJ, Ellis E, Hupp J, Tucker MR (2002) Contemporary oral and maxillofacial surgery. St. Louis: Elsevier Health Sciences, pp: 345-357.

9. Shatkin S, Hoffmeister FS (1965) Ameloblastoma: a rational approach to therapy. Oral Surg Oral Med Oral Pathol 20: 421-435.

10. Sehdev MK, Huvos AG, Strong EW, Gerold FP, Willis GW (1974)Ameloblastoma of maxilla and mandible. Cancer 33: 324-333.

11. Müller H, Slootweg PJ (1985) The ameloblastoma, the controversial approach to therapy. J Maxillofac Surg 13: 79-84.

12. Sitthiphan $P$, Jamayet NB, Srithavaj T, Tirasriwat A, Alam MK (2013) Mandibular defect rehabilitation with conventional removable partial denture: Case report. Int Med J 20: 618-620 